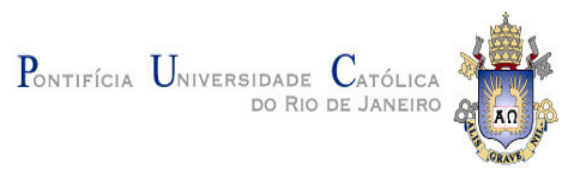

Rosana Lacerda Coelho Fernandes

\title{
APL de Mármore e Granito de Cachoeiro de Itapemirim: aprendizado por interação como fonte de inovações.
}

Dissertação de Mestrado

Dissertação apresentada como requisito parcial para obtenção do título de Mestre pelo Programa de PósGraduação em Engenharia Industrial da PUC-Rio.

Orientador: Paulo Roberto Tavares Dalcol 


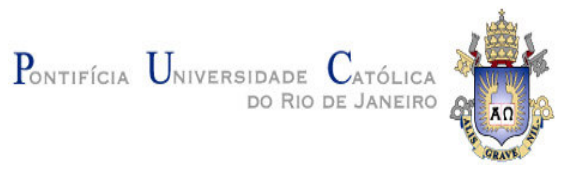

Rosana Lacerda Coelho Fernandes

\title{
APL de Mármore e Granito de Cachoeiro de Itapemirim: aprendizado por interação como fonte de inovações.
}

\begin{abstract}
Dissertação apresentada como requisito parcial para obtenção do título de Mestre pelo Programa de PósGraduação em Engenharia Industrial da PUC-Rio. Aprovada pela Comissão Examinadora abaixo assinada.
\end{abstract}

Paulo Roberto Tavares Dalcol Orientador PUC-Rio

Leila Cristina Nunes Gomes FMG Empreendimentos Hospitalares Ltda

Romeu e Silva Neto CEFET-Campos

José Eugenio Leal Coordenador Setorial do Centro Técnico Científico - PUC-Rio 
Todos os direitos reservados. É proibida a reprodução total ou parcial do trabalho sem autorização da universidade, da autora e do orientador.

\section{Rosana Lacerda Coelho Fernandes}

Engenheira Civil pela Universidade Federal do Espírito Santo (UFES) em 2001. Cursou Especialização em Gestão Industrial na FDV em parceria com IEL-ES, FINDES e SEBRAE em 2003.

Ficha Catalográfica

Fernandes, Rosana Lacerda Coelho

APL de mármore e granito de Cachoeiro de Itapemirim: aprendizado por interação como fonte de inovações / Rosana Lacerda Coelho Fernandes; orientador: Paulo Roberto Tavares Dalcol. - Rio de Janeiro: PUC, Departamento de Engenharia Industrial, 2006.

94 f. ; $30 \mathrm{~cm}$

Dissertação (mestrado) - Pontifícia Universidade Católica do Rio de Janeiro, Departamento de Engenharia Industrial

Inclui referências bibliográficas.

1. Engenharia industrial - Teses. 2. Arranjos produtivos locais. 3. Aprendizado por interação. 4. Inovações tecnológicas. I. Dalcol, Paulo Roberto Tavares. II. Pontifícia Universidade Católica do Rio de Janeiro. Departamento de Engenharia Industrial. III. Título. 


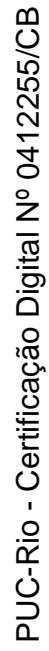

Ao meu esposo e aos meus pais em retribuição ao apoio e incentivo. 


\section{Agradecimentos}

Primeiramente, agradeço à Deus por estar ao meu lado em todo momento, me dando forças para suportar, principalmente, a ausência dos meus familiares.

Ao meu esposo por aceitar minha ausência durante o primeiro ano do mestrado e por sua paciência até aqui.

Aos meus pais pelo apoio e incentivo nessa busca pelo conhecimento.

Ao meu grande orientador professor Paulo Roberto Tavares Dalcol pela sua dedicação e por seus importantes comentários durante a condução deste trabalho.

À todos os professores e funcionários do DEI pelas contribuições e apoio.

Aos meus colegas de curso pela amizade e parceria ao longo dessa jornada.

À todos do APL de Mármore e Granito de Cachoeiro de Itapemirim-ES que dedicaram um pouco do seu tempo em entrevistas e que disponibilizaram os documentos que muito ajudaram para a realização desta dissertação. Em especial, que oferecer meus sinceros agradecimentos à Rogelio Paes Santos (Sebrae), Áureo Mameri (Sindirochas), Diti (Maqrochas), Luciano (Cetemag), Cristiano Sartório (GMC), Sergio Pedrosa (Cetemag), Prof. Tiago Bevilaqua (idealizador e $1^{\circ}$ coordenador do Rederochas), Sr. Pedro Zanivan (Pemagran) e Profeta (Cajugran).

À CAPES pelo apoio financeiro concedido para a realização deste programa de mestrado.

Não poderia deixar de agradecer também à minha tia Arite e aos meus primos Eduardo, Maria Helena, Flavia e Marcelo que me receberam em sua casa no início desta jornada.

Enfim, agradeço à Kamila, Larissa e Carla pela amizade e companheirismo durante o tempo em que fiquei no apartamento da Gávea. 


\section{Resumo}

Fernandes, Rosana Lacerda Coelho. APL de Mármore e Granito de Cachoeiro de Itapemirim: aprendizado por interação como fonte de inovações. Rio de Janeiro, 2006. 94p. Dissertação de Mestrado Departamento de Engenharia Industrial, Pontifícia Universidade Católica do Rio de Janeiro.

Com o declínio do sistema de produção em massa, o qual tinha um foco predominante nas grandes empresas, houve um redesenho das forças produtivas com tendências a uma estrutura que passou a favorecer as pequenas e médias empresas (PMEs), principalmente quando atuam de forma conjunta em aglomerações industriais e não isoladamente. Assim, foram surgindo, a partir dos anos 90, vários estudos apontando os chamados Arranjos Produtivos Locais (APLs) como uma forma de possibilitar a inserção competitiva das PMEs na economia moderna. Alguns pesquisadores consideram que as PMEs, quando inseridas nessas aglomerações, podem desenvolver uma dinâmica de aprendizado que leva a um processo de aquisição de conhecimentos. $\mathrm{O}$ aprendizado por interação, em particular, é apresentado como o principal responsável pela geração de inovações em PMEs. Dessa forma, esta dissertação buscou apresentar um estudo de caso, focando nas inovações geradas pelo aprendizado por interação no APL de Mármore e Granito de Cachoeiro de Itapemirim - ES, que se destaca como o principal produtor de rochas ornamentais do Brasil e como um dos maiores exportadores do mundo. Nesse APL foram encontradas duas principais fontes de aprendizado por interação: o GMC (Grupo de Melhoria Contínua) e o Empreender. O GMC, em especial, foi o foco de estudo deste trabalho, que buscou entender, principalmente, como ocorre a geração de inovações por meio da dinâmica de interação entre as empresas participantes desse grupo e entre este e as entidades que compõem a governança local, além de verificar alguns eventos relevantes de inovação do ponto de vista da empresa usuária/beneficiada.

\section{Palavras-chave}

Arranjos Produtivos Locais, Aprendizado por Interação, Inovações Tecnológicas. 


\section{Abstract}

Fernandes, Rosana Lacerda Coelho. APL de Mármore e Granito de Cachoeiro de Itapemirim: aprendizado por interação como fonte de inovações. Rio de Janeiro, 2006. 94p. Dissertação de Mestrado Departamento de Engenharia Industrial, Pontifícia Universidade Católica do Rio de Janeiro.

With the decline of the mass production system, which had a predominant focus on big companies, there was a readjustment of the productive forces with tendencies to a structure that started to favor the small and medium companies (SMCs), mainly when they act in an united way in industrial clusters and not separately. Starting from the nineties, several studies were developed pointing the calls clusters as a possibility for the competitive insertion of SMCs in the modern economy. Some researchers consider that SMCs, when inserted in clusters can develop a learning dynamic that leads to the process of acquisition of knowledge. The learning by interacting, in particular, is presented as the main responsible for the generation of innovations in SMCs. In that way, this dissertation presents a case study, focusing on the innovations generated by learning by interacting in the marble and granite cluster of Cachoeiro de Itapemirim, which stands out as the main producer of Brazil and as one of the larger exporters of the world. In this cluster two main learning by interacting sources were found: GMC (Group of Continuous Improvement) and Empreender. GMC, in especial, was the focus of this work, which sought to understand, mainly, how the generation of innovations occur through the interaction of the participant companies of that group and among these and the entities of the local governance, as well as to investigate some relevant events of innovation from the point of view of the companies.

\section{Keywords}

Industrial Clusters, Learning by Interacting, Technology Innovation. 


\section{Sumário}

1 Introdução

2 Aglomerações Industriais $\quad 21$

2.1. Origem da Discussão Sobre Aglomerações Industriais 21

2.2. Tipos de Aglomerações Industriais 23

2.2.1. Distritos Industriais Marshallianos 23

2.2.2. Millieu Innovateur 24

2.2.3. Cluster 26

2.3. Caracterização das Aglomerações Industriais no Brasil 27

2.4. Entendendo a Governança nos APLs 30

3 Aprendizado por Interação como Gerador de Inovações em APLs 34

3.1. Aprendizagem como Fonte de Inovações 34

3.2. Inovação e Sistemas de Inovação 38

3.2.1. Inovação 38

3.2.1. Sistemas de Inovação 39

3.3. O Papel da Inovação e do Processo Interativo de Aprendizado nos APLS

4 Descrição e Análise do APL de Mármore e Granito de Cachoeiro de Itapemirim - ES

4.1. Descrição do APL

4.2. Análise da Dinâmica de Funcionamento do APL

5 GMC: Gerador de Inovações Por Meio do Aprendizado Por Interação 58

5.1. Descrição da Proposta de Trabalho Apresentada ao MCT 58

5.2. Descrição da Implementação do Programa de Redes 61

5.3. Análise da Atuação do GMC Como Indutor de Inovações 64

5.4. Análise de Alguns Eventos Relevantes de Inovação do Ponto de Vista da Empresa Beneficiada/Usuária 
6 Considerações Finais

Referências Bibliográficas

84

Anexos 


\section{Lista de figuras}

Figura 1: Estrutura Metodológica da Dissertação. 17

Figura 2: Diferentes tipos de inovação. 38

Figura 3: Fluxos de Relacionamento em um Sistema Local de Inovação.41

Figura 4: Tipos de Interações. 44

Figura 5: Localização do APL de Rochas Ornamentais de Cachoeiro de Itapemirim no ES. 49

Figura 6: Principais Entidades do APL de Cachoeiro de Itapemirim - ES sob o ponto de vista da inovação: descrição e interações.

Figura 7: Estrutura, Dinâmica e Fontes de Interações do APL de Mármore 


\section{Lista de quadros}

Quadro 1: Definições e Principais Vantagens do Foco em Arranjos e Sistemas Produtivos Locais. 28

Quadro 2: Principais Características de Aglomerações com Governança Local de Grandes Empresas.

Quadro 3: Características de Aglomerações sem Governança Local Definida.

Quadro 4: Resumo das Respostas Sobre o Evento I. 70

Quadro 5: Resumo das Respostas Sobre o Evento II. 71

Quadro 6: Resumo das Respostas Sobre o Evento III. 72

Quadro 7: Resumo das Respostas Sobre o Evento IV. 73

Quadro 8: Resumo das Respostas Sobre o Evento V. 74

Quadro 9: Resumo das Respostas Sobre o Evento VI. 75

Quadro 10: Resumo das Respostas Sobre as Contribuições do GMC. 76 


\section{Lista de siglas}

ACISCI - Associação Comercial, Industrial e de Serviços de Cachoeiro de Itapemirim.

APL - Arranjo Produtivo Local.

BANDES - Banco de Desenvolvimento do Espírito Santo.

CETEM - Centro de Tecnologia Mineral.

CETEMAG - Centro Tecnológico do Mármore e Granito.

CNPq - Conselho Nacional de Desenvolvimento Científico e Tecnológico.

CREDIROCHAS - Cooperativa de Economia e Crédito Mútuo dos Proprietários

da Indústria de Rochas Ornamentais, Cal e Calcários do Espírito Santo.

FINEP - Financiadora de Estudos e Projetos.

FNDCT - Fundo Nacional de Desenvolvimento Científico e Tecnológico.

GEOR - Gestão Orientada para Resultados.

GMC - Grupo de Melhoria Contínua.

ICMS - Imposto sobre Circulação de Mercadorias e Prestação de Serviços

IEL-ES - Instituo Euvaldo Lodi do Espírito Santo.

MAQROCHAS - Associação dos Fabricantes de Máquinas e Equipamentos do Setor de Rochas Ornamentais.

MCT - Ministério da Ciência e Tecnologia.

MDIC - Ministério do Desenvolvimento Indústria e Comércio.

PMEs - Pequenas e Médias Empresas.

REDEROCHAS - Rede do Setor de Rochas Ornamentais.

Redesist - Rede de Pesquisa em Sistemas Produtivos e Inovativos Locais.

SEBRAE - Serviço de Apoio às Micro e Pequenas Empresas. 
SINDIMÁRMORE - Sindicato dos Trabalhadores na Indústria do Mármore, Granito e Calcário do Espírito Santo.

SINDIROCHAS - Sindicato da Indústria de Rochas Ornamentais do Espírito Santo.

SLI - Sistema Local de Inovação.

SNI - Sistema Nacional de Inovação.

SPIL - Sistema Produtivo e Inovativo Local.

UFES - Universidade Federal do Espírito Santo. 